Review began 12/29/2021 Review ended 01/10/2022 Published 01/11/2022

๑) Copyright 2022 Alvarez-Payares et al. This is an open access article distributed under the terms of the Creative Commons Attribution License CC-BY 4.0., which permits unrestricted use, distribution, and reproduction in any medium, provided the original author and source are credited.

\section{Clindamycin Efficacy in Patients With Methicillin-Sensitive Staphylococcus aureus in a Fourth-Level Hospital in the City of Medellín}

Jose C. Alvarez-Payares ${ }^{1}$, Jair E. Palacios $\mathrm{M}^{2}$, Edwin De la Peña ${ }^{3}$, Huxdley B. Cabrera ${ }^{2}$, Santiago GiraldoRamírez $^{2}$, Marcela Loaiza ${ }^{3}$, Fabian Jaimes ${ }^{2}$, Joaquin Rodelo ${ }^{2}$, Jose Ágamez-Gómez ${ }^{3}$

1. Departamento de Medicina Interna, Universidad de Antioquia, Medellín, COL 2. Departamento de Medicina Interna, Hospital Universitario San Vicente Fundación, Medellín, COL 3. Facultad de Medicina, Universidad de Antioquia, Medellín, COL

Corresponding author: Jose C. Alvarez-Payares, josecarlosalvarezj9@hotmail.com

\title{
Abstract
}

Background: The antibiotic of choice for methicillin-sensitive Staphylococcus aureus (MSSA) bacteremia is antistaphylococcal penicillins, such as oxacillin, but cefazolin has also risen as an equally effective alternative. Murine models have suggested that clindamycin is a therapeutic alternative for Staphylococcus aureus bacteremia (SAB).

Methods: In this retrospective cohort study, patients from the Hospital Universitario San Vicente Fundación (HUSVF) in Medellín, Colombia, were recruited from January 2013 and December 2019. Patients with positive blood culture for MSSA, with at least one follow-up blood culture, and those with more than 72 hours of parenteral antibiotic therapy for SAB were selected. The main objective was to determine the efficacy of clindamycin compared to other antibiotics to achieve a microbiologic cure. Secondary results included in-hospital mortality and hospital stay.

Results: A total of 486 patients were included (clindamycin $=50$ and other anti-MSSA $=436$ ). The patients in the clindamycin group had a lower rate of microbiological cure $(n=41$ [84\%]) compared to other antibiotics $(\mathrm{n}=367$ [84\%]) (OR 1.08 IC 95\% 0.74-1.58). In secondary outcomes, no statistically significant differences were observed in the in-hospital mortality. The main source of SAB was a central or peripheral catheter (58\%).

Conclusions: Our study found no differences in the rate of microbiological cure, in-hospital mortality, and hospital stay on the clindamycin group compared to other anti-MSSA antibiotics. However, in patients with metastatic complications, the rate of microbiological cure is reduced, and the in-hospital mortality is higher in patients with more severe disease.

Categories: Internal Medicine, Medical Education, Infectious Disease

Keywords: treatment choices, bloodstream infections, clindamycin, mssa bacteremia, methicillin-sensitive staphylococcus aureus

\section{Introduction}

Staphylococcus aureus (SA) is one of the most frequent causes of bacteremia in the hospitals of the United States $[1,2]$. It is a public health concern, associated with a mortality rate of $20 \%-30 \%$ in adults and $5 \%$ in children [3]. Historically, antibiotics of choice for methicillin-sensitive Staphylococcus aureus (MSSA) have been semisynthetic penicillins. Nonetheless, the treatment is complex due to the virulence, antibiotic resistance, high frequency of therapeutic failure, and scarce alternative treatment [2]. This has led to the search for new management options.

In toxin-mediated SA infections, such as necrotizing fasciitis, some guidelines recommend adding clindamycin to the standard treatment. These recommendations are based on expert opinions with limited clinical evidence $[4,5]$. Animal studies and human observational studies suggest the benefit from clindamycin $[6,7]$; however, no clinical trials sustain this strategy in SA bacteremia (SAB).

Concern surrounds the clindamycin's efficacy, specifically in a bacteremia scenario - whether it is related to its bacteriostatic effect or not. Furthermore, clinical evidence supporting its use in this context is scarce [3]. Hence, the objective of the present study was to determine the efficacy of clindamycin treatment compared with other antibiotics in patients with SAB diagnosis in a fourth-level hospital.

\section{Materials And Methods}

\section{Study design and population}


This is a historical cohort study. Patients who were cared for at the Hospital Universitario San Vicente Fundación (HUSVF) in Medellín, Colombia, from January 2013 to December 2019 were recruited. The study was approved by the hospitals' Ethics Committee and Investigation Directorate, and the approval number was 24-2019.

\section{Patients}

Patients who were 18 years or older with a positive MSSA blood culture, at least one follow-up blood culture, and at least 72 hours of parenteral antibiotic therapy were included. Patients with SAB diagnosis in the 30 days before hospitalization, combined antibiotic therapy or polymicrobial cultures, were excluded.

\section{Variables}

Patients were defined as exposed to clindamycin when parenteral antibiotic treatment was administered for at least 72 hours. Non-exposed patients were defined as those who received other anti-MSSA antibiotics (oxacillin, ceftriaxone, cefazolin, piperacillin/tazobactam, and ampicillin/sulbactam).

The primary outcome was a microbiological cure, which is defined as the absence of bacteria in control blood culture after 72 hours of anti-MSSA-directed therapy [8]. Secondary outcomes were as follows: (1) Hospital stay measured as days after the first positive blood culture up to discharge in survivors and (2) in-hospital mortality.

Confounding variables were selected based on the available literature, such as age, severity based on Sequential Organ Failure Assessment Score (SOFA) [9]. In patients for whom information was not available, severity was assessed according to ICU admission, immunosuppression (immunocompromise is defined as neutropenia $\leqslant 500$ cells $/ \mathrm{mm}^{3}$, systemic steroid use for more than a month, transplant patients, biologic drug use, or cancer chemotherapy), lactate $>2 \mathrm{mmol} / \mathrm{L}$, and metastatic complications $[8,10,11]$.

\section{Data source}

Patients were drawn from MSSA positive blood cultures from the time frame previously described. The electronic health record was reviewed to evaluate the demographical and clinical characteristics as well as the antibiotic treatment. Data were collected from January 2020 to February 2021.

\section{Bias control}

An information bias was identified, and it was mitigated through the definition of minimum variables of every patient to be included in the database, during the study period.

\section{Sample size}

No formal sample size calculation was performed as the entire population available in the cohort was analyzed.

\section{Statistical analysis}

Microbiological cure and in-hospital mortality were compared between groups (patients treated with clindamycin and patients treated with other antibiotics) through the chi-square test of independence or Fisher's exact test according to the expected value in the cell. In-patient stance days were compared through the Mann-Whitney U test. For an adjusted analysis of microbiological cure and in-hospital mortality, according to the confounding variables previously defined, multivariable logistic analyses were performed with previous validation of the assumptions of the absence of multicollinearity, absence of interaction, and absence of linearity among continuous independent variables and the respective outcome logit. Results are shown as odds ratio (OR) with a 95\% confidence interval (CI).

\section{Results}

\section{Patients}

A total of 1126 charts from patients with SAB (including MSSA and MRSA) were reviewed, from a fourthlevel hospital in Medellín, in the time-lapse of January 2013 and December 2018. After inclusion and exclusion criteria were applied, 486 patients with MSSA bacteremia were obtained, with 50 in the clindamycin group and 436 in the other antibiotics group (Figure 1). The information was collected from January 2020 to February 2021. 


\section{Cureus}

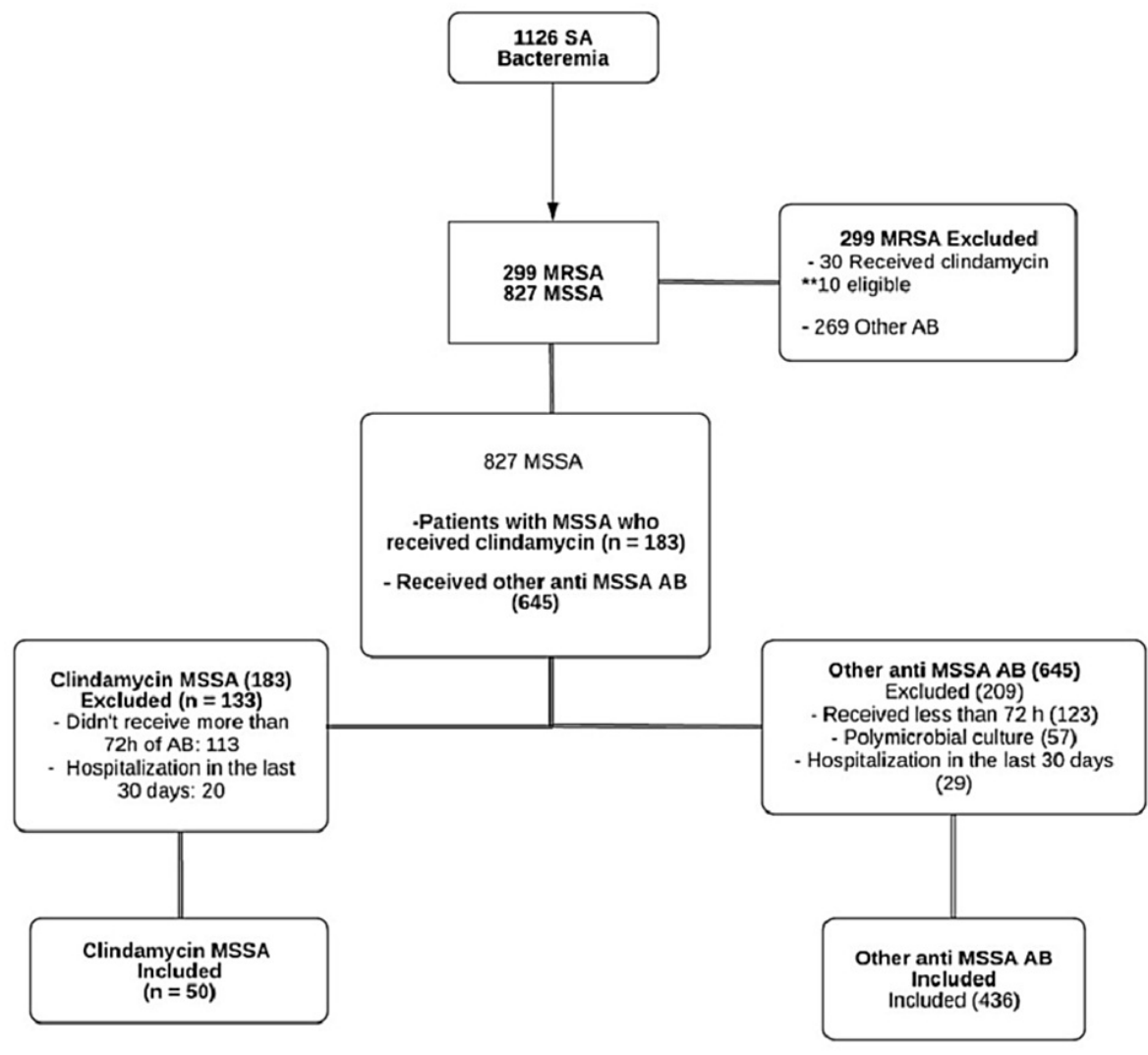

FIGURE 1: Recruitment, assignation, and inclusion in the primary analysis

SA, Staphylococcus aureus; MRSA, methicillin-resistant Staphylococcus aureus; MSSA, methicillinsensitive Staphylococcus aureus.

\section{Patients' characteristics}

The mean age was 53.5 years $( \pm 17.9)$, predominantly male $(58 \%)$ and mestizo $(87 \%)$. The most frequent comorbidities were advanced chronic kidney disease (49\%), diabetes mellitus (27\%), and cancer (10\%). The main source of SAB was a central or peripheral catheter (58\%). One-third of the patients had lactate levels > $2 \mathrm{mmol} / \mathrm{L}$, and a fifth required admission to the intensive care unit. In the group of other antibiotics with MSSA coverage, $50 \%$ received ceftriaxone, and $26.5 \%$ received cefazolin; most of the time, patients received empirical therapy (82\%), mainly with vancomycin. The patients in the clindamycin group (88.25\%) received an IV dose of $900 \mathrm{mg}$ TID. The most common metastatic complications were pulmonary (21.57\%) and osteomuscular ones (17.91\%) (Table 1).

\begin{tabular}{llll} 
Variables & Clindamycin, $\mathbf{N}=\mathbf{5 0}(\mathbf{1 0 . 2 8} \%)$ & No clindamycin, $\mathbf{N}=\mathbf{4 3 6}(\mathbf{8 9 . 7 1 \% )}$ & p values \\
\hline Demographical & & & 0.052 \\
Age, Mean \pm SD & $56.5 \pm 17.4$ & $52.6 \pm 18.1$ & 0.446 \\
Sex $\mathrm{n}(\%)$, Male & $31(62 \%)$ & $254(58 \%)$ & $<0.001$ \\
Comorbidities & & & $<0.001$ \\
Immunosuppression, any cause $\mathbf{n}(\%)$ & $1(2 \%)$ & $8(2 \%)$ & $<0.001$ \\
Neutropenia < 500 & $4(8 \%)$ & $52(12 \%)$ & 0.015 \\
Systemic steroid use for more than a month & $0(0 \%)$ & $13(3 \%)$ & $20(5 \%)$ \\
Transplant & $1(2 \%)$ & &
\end{tabular}




\section{Cureus}

\begin{tabular}{|c|c|c|c|}
\hline Cirrhosis & $5(10 \%)$ & $14(3 \%)$ & 0.003 \\
\hline Cancer & $5(10 \%)$ & $35(8 \%)$ & 0.015 \\
\hline HIV with CD4 < 200 & $3(6 \%)$ & $3(0.7 \%)$ & 0.002 \\
\hline Advanced CKD & $16(32 \%)$ & $227(52 \%)$ & 0.019 \\
\hline \multicolumn{4}{|l|}{ CV disease } \\
\hline $\mathrm{CHF}$ & $17(34 \%)$ & $54(12 \%)$ & 0.019 \\
\hline Diabetes mellitus & $11(22 \%)$ & $115(26 \%)$ & 0.549 \\
\hline \multicolumn{4}{|l|}{ Primary source of SA bacteremia } \\
\hline Unknown & $5(10 \%)$ & $42(10 \%)$ & $<0.001$ \\
\hline Central/peripheral catheter & $32(64 \%)$ & $240(55 \%)$ & $<0.001$ \\
\hline Abscess & $2(4 \%)$ & $28(6 \%)$ & 0.666 \\
\hline Cellulitis & $7(14 \%)$ & $10(2 \%)$ & $<0.001$ \\
\hline SOFA score & $1(0-2)$ & $4(1-6)$ & $<0.001$ \\
\hline Lactate $>2 \mathrm{mmol} / \mathrm{L}$ & $10(23 \%)$ & $142(34 \%)$ & 0.039 \\
\hline Vasopressor/inotrope use & $4(8 \%)$ & $49(11 \%)$ & 0.109 \\
\hline Ventilatory support & $5(10 \%)$ & $49(11 \%)$ & 0.243 \\
\hline ICU requirement & $6(12 \%)$ & $96(22 \%)$ & 0.211 \\
\hline Empirical antibiotic & $41(82 \%)$ & $346(79 \%)$ & 0.003 \\
\hline Empirical treatment duration (days) & $3(2-3)$ & $3(2-3)$ & 0.449 \\
\hline Directed therapy duration (days) & $14(10-16)$ & $14(9-17)$ & 0.002 \\
\hline \multicolumn{4}{|l|}{ Metastasis complications } \\
\hline Endocarditis & 0 & $34(8 \%)$ & 0.010 \\
\hline \multicolumn{4}{|l|}{ Pulmonary } \\
\hline Pneumonia & $9(18 \%)$ & 70 (16\%) & $<0.001$ \\
\hline \multicolumn{4}{|l|}{ Osteomuscular } \\
\hline Osteomyelitis & $10(20 \%)$ & $58(13 \%)$ & $<0.001$ \\
\hline Septic arthritis & 0 & $8(2 \%)$ & $<0.001$ \\
\hline Skin & $4(4 \%)$ & $31(7.11 \%)$ & \\
\hline Cellulitis & $3(6 \%)$ & $17(4 \%)$ & 0.010 \\
\hline Abscesses & 0 & $28(6.4 \%)$ & 0.160 \\
\hline CNS & $1(0.9 \%)$ & $13(3 \%)$ & \\
\hline
\end{tabular}

\section{TABLE 1: Patient's characteristics}

CV, Cardiovascular; CNS, central nervous system; CHF, congestive heart failure; CKD, chronic kidney disease defined as an eGFR < $30 \mathrm{~mL} / \mathrm{min}$; eGFR, estimated glomerular filtration rate.

\section{Main results}

The group of patients treated with clindamycin for SAB achieved a microbiological cure in $82 \%$ of cases $(\mathrm{n}=$ 41) compared to $84 \%(n=367)$ in the group of other antibiotics (OR 1.08 IC $95 \% 0.74-1.58)$. In secondary outcomes, no statistically significant differences in the in-hospital mortality were found (Figure 2); however, a significant difference in the metastatic complications was observed (20\% vs $26 \%)$. All the clinical outcomes are highlighted in Table 2. 


\section{Cureus}

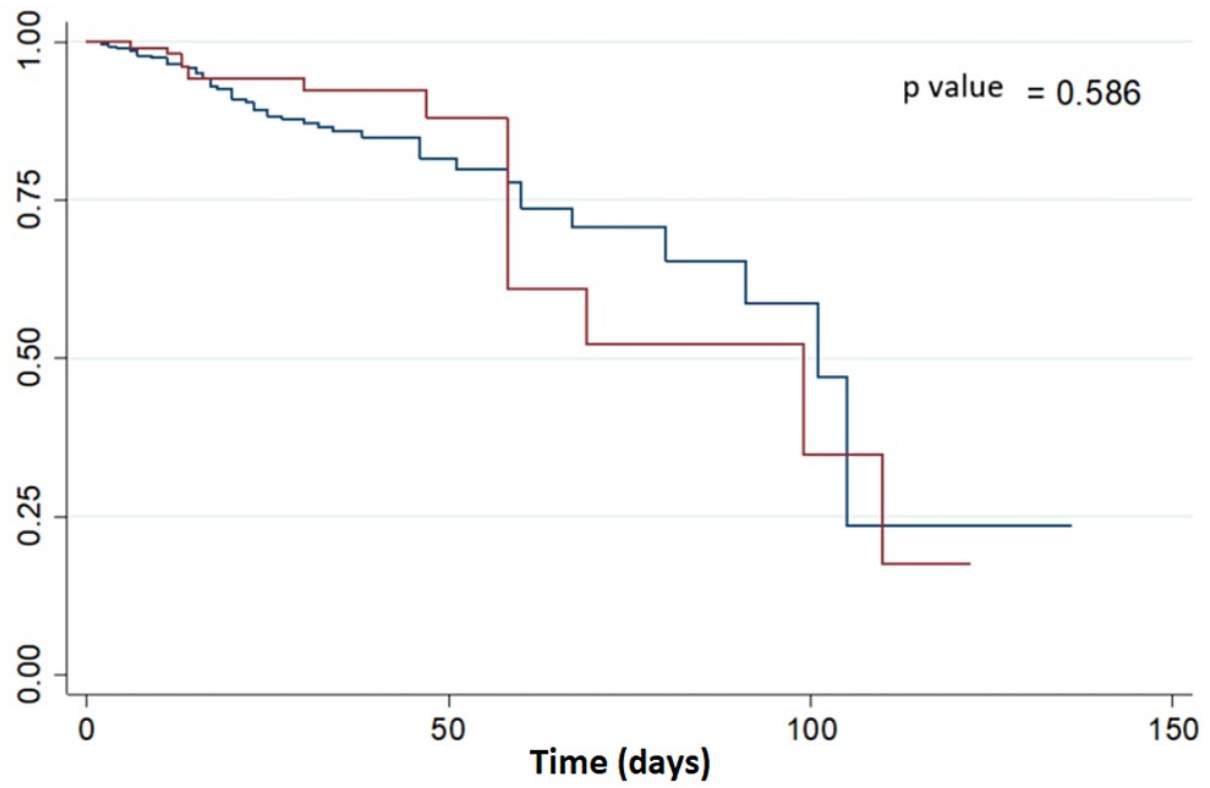

Other antibiotics

Clindamycin

FIGURE 2: Survival curve of patients with MSSA bacteremia treated with clindamycin or other antibiotics

MSSA, Methicillin-sensitive Staphylococcus aureus.

\begin{tabular}{|c|c|c|c|}
\hline Outcome & Clindamycin, $\mathrm{N}=50(10.28 \%)$ & No clindamycin, N = 436 (89.71\%) & $p$ value \\
\hline \multicolumn{4}{|l|}{ Primary } \\
\hline Microbiological cure & $41(82 \%)$ & $367(84 \%)$ & $<0.001$ \\
\hline \multicolumn{4}{|l|}{ Secondary } \\
\hline In-hospital mortality & $15(14 \%)$ & $56(13 \%)$ & 0.851 \\
\hline Stay hospital (days) & $28(17-42)$ & $21(15-36)$ & 0.011 \\
\hline Persistant bacteremia & $10(20 \%)$ & $113(26 \%)$ & 0.1 \\
\hline Microbiological clearance time (days) & $3(3-4)$ & $3(3-5)$ & 0.078 \\
\hline Metastatic complications & $13(26 \%)$ & $142(33 \%)$ & $<0.001$ \\
\hline
\end{tabular}

TABLE 2: Primary and secondary outcomes

\section{Multivariate analysis}

After the multivariate analysis was performed, with the confounding variables previously established in the protocol, an OR of 1.45 (CI 95\% 0.88-2.39) for the microbiological cure and 0.74 (CI 95\% 0.36-1.54) for inhospital mortality were found (Tables 3,4 ). 


\section{Cureus}

\begin{tabular}{|c|c|c|c|c|}
\hline Variables & OR & Cl 95\% & Adjusted OR & Adjusted $\mathrm{Cl} 95 \%$ \\
\hline Anti-MSSA antibiotics other than clindamycin & 1.08 & $0.74-1.58$ & 1.45 & $0.88-2.39$ \\
\hline Age & 0.99 & $0.97-1.00$ & 0.99 & $0.98-1.00$ \\
\hline Immunocompromise & 1.40 & $0.76-2.57$ & 1.90 & $0.90-4.01$ \\
\hline Lactate $>2$ mmol/L & 0.26 & $0.16-0.42$ & 0.55 & $0.29-1.04$ \\
\hline ICU admission & 0.22 & $0.13-0.37$ & 0.63 & $0.31-1.29$ \\
\hline SOFA score & 0.78 & $0.73-0.84$ & 0.85 & $0.76-0.94$ \\
\hline Metastatic complications & 0.22 & $0.13-0.36$ & 0.27 & $0.15-0.51$ \\
\hline
\end{tabular}

\section{TABLE 3: Association between MSSA bacteremia treated with clindamycin or other antibiotics with microbiological cure after multivariate analysis}

Immunocompromise is defined as neutropenia $\leq 500$ cells $/ \mathrm{mm}^{3}$, systemic steroid use for more than a month, transplant patients, biologic drug use, or cancer chemotherapy.

MSSA, Methicillin-sensitive Staphylococcus aureus; SOFA, Sequential Organ Failure Assessment Score.

\begin{tabular}{|c|c|c|c|c|}
\hline Variables & OR & Cl 95\% & Adjusted OR & Adjusted $\mathrm{Cl} 95 \%$ \\
\hline Anti-MSSA antibiotics other than clindamycin & 1.15 & $0.71-1.87$ & 0.74 & $0.36-1.54$ \\
\hline Age & 1.03 & $1.01-1.04$ & 1.04 & $1.01-1.06$ \\
\hline Immunocompromise & 1.38 & $0.76-2.50$ & 2.12 & $0.95-4.76$ \\
\hline Lactate $>2 \mathrm{mmol} / \mathrm{L}$ & 9.35 & $5.01-17.43$ & 3.92 & $1.61-9.55$ \\
\hline ICU admission & 9.74 & $5.41-17.55$ & 3.47 & $1.58-7.62$ \\
\hline SOFA score & 1.48 & $1.35-1.63$ & 1.26 & $1.13-1.41$ \\
\hline Metastatic complications & 2.92 & $1.69-5.05$ & 1.96 & $0.92-4.21$ \\
\hline
\end{tabular}

TABLE 4: Association between MSSA bacteremia treated with clindamycin or other antibiotics with in-hospital mortality after multivariate analysis

Immunocompromise is defined as neutropenia $\leq 500$ cells $/ \mathrm{mm}^{3}$, systemic steroid use for more than a month, transplant patients, biologic drug use, or cancer chemotherapy.

MSSA, Methicillin-sensitive Staphylococcus aureus; SOFA, Sequential Organ Failure Assessment Score.

\section{Discussion}

Adequate antibiotic therapy is fundamental to prevent adverse outcomes associated with SAB [1]; therefore, the selection of an antimicrobial agent is a critical step. In our institution, the use of clindamycin as monotherapy in SAB has been driven by local studies in murine models that support its use in severe infections. In a neutropenic murine model, it was observed that clindamycin had bactericidal activity and the capacity to achieve a drop of more than three logs of colony-forming units (CFU), which equates to a bacterial inoculum $>99.9 \%$, indicating a potent in vivo bactericidal effect [7].

We believe that this is the biggest cohort published until this date evaluating clindamycin's role for SAB treatment. In this retrospective cohort, we found no statistically significant difference favoring in relation to microbiological cure, even when confounding factors were adjusted. As no similar study testing clindamycin's efficacy in SAB treatment in humans has been reported, our results cannot be contrasted with other clinical studies. Nevertheless, the microbiological cure rate with clindamycin differs from those reported In the literature for ceftriaxone, cefazolin, and nafcillin/oxacillin (93.1\%, 87.2\%, and 91.2\%, respectively) $[12,13]$. 
On multivariate analysis, we found that the primary outcome was reduced on patients in the clindamycin group with higher SOFA scores and metastatic complications. This suggests that clindamycin may not be a good alternative in patients with severe infections and complicated bacteremia.

Stay hospital and in-hospital mortality were similar in both groups; even after confounding factors were analyzed, no differences were observed (OR 0.74, IC 95\% 0.36-1.54). There are some factors related to this outcome, such as one reported by a prospective multicentric European study of 987 patients, in which the persistence of SAB, defined in different times, was independently associated with mortality ( $\geqslant 2$ days, HR 1.93; IC 95\% 1.51-2.46) [8]. In our study, no significant difference in the microbiological clearance time was observed, which was higher than three days in both groups; nonetheless, one of the limitations of the present study was that the first control blood culture was taken at 72 hours from the first one.

Additionally, factors such as age, blood lactate $>2 \mathrm{mmol} / \mathrm{L}$, ICU admission, and SOFA score were associated with increased mortality as was previously described in other studies [9,14-17]. Immunocompromised state and metastatic complications were not associated with higher mortality. The previous retrospective analysis did not identify immunocompromise as a mortality risk factor in SAB [18], although some studies did show an association [19-20]. Another important aspect is that it is known that endocarditis and pulmonary infection sources increase mortality, but no representative sample was found in neither group [18].

This study has some limitations such as its retrospective nature, conditioning a risk for incomplete information, the incapacity to determine all the confounding factors that may have a potential impact on treatment failure, e.g., control blood cultures were not taken daily, which can lead to uncertainty about the real duration of bacteremia. This has a direct impact on mortality rates [8]. Despite these limitations, this is a rare study comparing clindamycin with other antibiotics in MSSA bacteremia. Further studies are required to determine if clindamycin is equally effective as other anti-MSSA antibiotics in SAB treatment regarding microbiological cure, metastatic complications, and death. Even though the standard treatment for MSSA bacteremia is antistaphylococcal beta-lactams such as oxacillin or cefazolin, evidence is deficient and is based on observational studies [2].

\section{Conclusions}

This is a rare study to explore the use of clindamycin for SAB. Our results suggest that different antistaphylococcal antibiotics for SAB treatment regarding microbiologic cure and intra-hospital mortality may be an option. However, in patients with metastatic complications, microbiological cure in the clindamycin group was inferior compared to other antibiotics. The differences in comorbidities, the type of study, and the patient's sample may explain the results; hence, further studies, ideally prospective ones, are required to evaluate the clindamycin's role in this scenario.

\section{Additional Information \\ Disclosures}

Human subjects: Consent was obtained or waived by all participants in this study. Hospital Universitario San Vicente Fundación issued approval 24-2019. The study was approved by the hospital's Ethics Committee and Investigation Directorate. Animal subjects: All authors have confirmed that this study did not involve animal subjects or tissue. Conflicts of interest: In compliance with the ICMJE uniform disclosure form, all authors declare the following: Payment/services info: All authors have declared that no financial support was received from any organization for the submitted work. Financial relationships: All authors have declared that they have no financial relationships at present or within the previous three years with any organizations that might have an interest in the submitted work. Other relationships: All authors have declared that there are no other relationships or activities that could appear to have influenced the submitted work.

\section{References}

1. Naber CK: Staphylococcus aureus bacteremia: epidemiology, pathophysiology, and management strategies . Clin Infect Dis. 2009, 48:S231-7. 10.1086/598189

2. Holland TL, Arnold C, Fowler VG Jr: Clinical management of Staphylococcus aureus bacteremia: a review . JAMA. 2014, 312:1330-41. 10.1001/jama.2014.9743

3. Martínez-Aguilar G, Hammerman WA, Mason EO Jr, Kaplan SL: Clindamycin treatment of invasive infections caused by community-acquired, methicillin-resistant and methicillin-susceptible Staphylococcus aureus in children. Pediatr Infect Dis J. 2003, 22:593-8. 10.1097/01.inf.0000073163.37519.ee

4. Brown NM, Brown EM: Treatment of methicillin-resistant Staphylococcus aureus (MRSA): updated guidelines from the UK. J Antimicrob Chemother. 2021, 76:1377-8. 10.1093/jac/dkab036

5. Gillet Y, Dumitrescu O, Tristan A, et al.: Pragmatic management of Panton-Valentine leukocidin-associated staphylococcal diseases. Int J Antimicrob Agents. 2011, 38:457-64. 10.1016/j.ijantimicag.2011.05.003

6. Campbell AJ, Dotel R, Blyth CC, Davis JS, Tong SY, Bowen AC: Adjunctive protein synthesis inhibitor antibiotics for toxin suppression in Staphylococcus aureus infections: a systematic appraisal. J Antimicrob Chemother. 2019, 74:1-5. 10.1093/jac/dky387

7. Zuluaga AF, Agudelo M, Rodriguez CA, Vesga O: In vivo efficacy of four generic products of clindamycin compared with the original compound in the neutropenic mouse thigh infection model. Int J Antimicrob Ag. 
2007, 1-2. 10.13140/2.1.3365.4088

8. Kuehl R, Morata L, Boeing C, et al.: Defining persistent Staphylococcus aureus bacteraemia: secondary analysis of a prospective cohort study. Lancet Infect Dis. 2020, 20:1409-17. 10.1016/S1473-3099(20)30447-3

9. Guimaraes AO, Cao Y, Hong K, et al.: A prognostic model of persistent bacteremia and mortality in complicated Staphylococcus aureus bloodstream infection. Clin Infect Dis. 2019, 68:1502-11.

10.1093/cid/ciy739

10. Ferreira FL, Bota DP, Bross A, Mélot C, Vincent JL: Serial evaluation of the SOFA score to predict outcome in critically ill patients. JAMA. 2001, 286:1754-8. 10.1001/jama.286.14.1754

11. Hill PC, Birch M, Chambers S, et al.: Prospective study of 424 cases of Staphylococcus aureus bacteraemia: determination of factors affecting incidence and mortality. Intern Med J. 2001, 31:97-103. 10.1111/j.14440903.2001.00029.x

12. Hamad Y, Connor L, Bailey TC, George IA: Outcomes of outpatient parenteral antimicrobial therapy with ceftriaxone for methicillin-susceptible Staphylococcus aureus bloodstream Infections-a single-center observational study. Open Forum Infect Dis. 2020, 7:ofaa341. 10.1093/ofid/ofaa341

13. Twilla JD, Algrim A, Adams EH, Samarin M, Cummings C, Finch CK: Comparison of nafcillin and cefazolin for the treatment of methicillin-susceptible Staphylococcus aureus bacteremia. Am J Med Sci. 2020, 360:3541. 10.1016/j.amjms.2020.04.006

14. López-Cortés LE, Gálvez-Acebal J, Rodríguez-Baño J: Therapy of Staphylococcus aureus bacteremia: evidences and challenges. Enferm Infecc Microbiol Clin (Engl Ed). 2020, 38:489-97. 10.1016/i.eimc.2020.01.018

15. Jensen AG, Wachmann CH, Espersen F, Scheibel J, Skinhøj P, Frimodt-Møller N: Treatment and outcome of Staphylococcus aureus bacteremia: a prospective study of 278 cases. Arch Intern Med. 2002, 162:25-32. 10.1001/archinte.162.1.25

16. Abbas M, Rossel A, de Kraker ME, et al.: Association between treatment duration and mortality or relapse in adult patients with Staphylococcus aureus bacteraemia: a retrospective cohort study. Clin Microbiol Infect. 2020, 26:626-31. 10.1016/..cmi.2019.07.019

17. van Hal SI, Jensen SO, Vaska VL, Espedido BA, Paterson DL, Gosbell IB: Predictors of mortality in Staphylococcus aureus bacteremia. Clin Microbiol Rev. 2012, 25:362-86. 10.1128/CMR.05022-11

18. Sasson G, Bai AD, Showler A, et al.: Staphylococcus aureus bacteremia in immunosuppressed patients: a multicenter, retrospective cohort study. Eur J Clin Microbiol Infect Dis. 2017, 36:1231-41. 10.1007/s10096017-2914-y

19. Kaech C, Elzi L, Sendi P, Frei R, Laifer G, Bassetti S, Fluckiger U: Course and outcome of Staphylococcus aureus bacteraemia: a retrospective analysis of 308 episodes in a Swiss tertiary-care centre. Clin Microbiol Infect. 2006, 12:345-52. 10.1111/j.1469-0691.2005.01359.x

20. Greenberg JA, David MZ, Hall JB, Kress JP: Immune dysfunction prior to Staphylococcus aureus bacteremia is a determinant of long-term mortality. PLoS One. 2014, 9:e88197. 10.1371/journal.pone.0088197 